\title{
Publisher Correction: Vimentin knockout results in increased expression of sub-endothelial basement membrane components and carotid stiffness in mice
}

\author{
Benoit Langlois ${ }^{1}$, Ekaterina Belozertseva ${ }^{1}$, Ara Parlakian ${ }^{2}$, Mustapha Bourhim $^{1}$, \\ Jacqueline Gao-Li' ${ }^{2}$, Jocelyne Blanc ${ }^{2}$, LeiTian ${ }^{2}$, Dario Coletti ${ }^{2}$, Carlos Labat ${ }^{1}$, \\ Zhor Ramdame-Cherif ${ }^{1}$, Pascal Challande ${ }^{3}$, Véronique Regnault ${ }^{1}$, Patrick Lacolley ${ }^{1}$ \& Zhenlin Li $\oplus^{2}$ \\ Correction to: Scientific Reports https://doi.org/10.1038/s41598-017-12024-z, published online 14 September \\ 2017
}

The original version of this Article contained typographical errors in the spelling of the authors Zhenlin Li and Patrick Lacolley, which were incorrectly given as Li Zhenlin and Patrick lacolley respectively. These errors have now been corrected in the PDF and HTML versions of the Article.

(i) Open Access This article is licensed under a Creative Commons Attribution 4.0 International (c) License, which permits use, sharing, adaptation, distribution and reproduction in any medium or format, as long as you give appropriate credit to the original author(s) and the source, provide a link to the Creative Commons license, and indicate if changes were made. The images or other third party material in this article are included in the article's Creative Commons license, unless indicated otherwise in a credit line to the material. If material is not included in the article's Creative Commons license and your intended use is not permitted by statutory regulation or exceeds the permitted use, you will need to obtain permission directly from the copyright holder. To view a copy of this license, visit http://creativecommons.org/licenses/by/4.0/.

(C) The Author(s) 2018

\footnotetext{
${ }^{1}$ Inserm, UMR_S 1116, Université de Lorraine, Nancy, France. ${ }^{2}$ Sorbonne Universités, UPMC Univ Paris 06, INSERM ERL U1164, Institut Biologie Paris-Seine, Paris, CNRS, UMR 8256, France. ${ }^{3}$ Sorbonne Universités, UPMC Univ Paris 06, Institut Jean Le Rond d'Alembert, Paris, CNRS, UMR 7190, France. Benoit Langlois and Ekaterina Belozertseva contributed equally to this work. Correspondence and requests for materials should be addressed to Z.L. (email: zhenlin.li@upmc.fr)
} 